\title{
Differences between family doctors and dermatologists in the diagnosis of potentially transmissible skin diseases among pool swimmers
}

\begin{abstract}
Introduction: Due to the difficult financial conditions in Greece, most of the prospective swimmers do not go to the dermatologist for their medical report regarding transmissible dermatological diseases. They receive this report from their family doctors. The purpose of this study was the evaluation of the ability of other specialties on recognizing and diagnosing transmissible dermatological diseases of swimmers.
\end{abstract}

Methods: A total of 1090 people were examined. The first group consisted of 479 people who were examined for the first time in the medical office of the swimming pool and had no medical report about a transmissible dermatological disease. The second group consisted of the remaining 611 people, who had a recent medical report from a family doctor certifying that they did not suffer from a transmissible dermatological disease.

Results: The $11.7 \%$ of the first group had some potentially transmissible dermatological disease, while the corresponding percentage of the second group was $5.7 \%$. Virtually one in two swimmers of the second group suffered from a potentially transmissible dermatological disease and had been diagnosed as healthy by a family doctor.

Conclusions: There are issues with the diagnosis of dermatological diseases by nondermatologists, since some lesions might not be recognized by General Practitioners or family doctors. The reason for that might be the difficulty in the diagnoses of the dermatological diseases, the training of other specialties in dermatological lesions and the financial status of the people examined.

Keywords: dermatology, swimming pools, public health, mycoses, warts, family doctors
Volume 4 Issue 2 - 2020

\author{
Papadopoulos lordanis \\ Department of Aesthetic and Cosmetology, Alexander \\ Technological Educational Institute of Thessaloniki, Greece \\ Correspondence: Papadopoulos lordanis, Department \\ of Aesthetic and Cosmetology, Alexander Technological \\ Educational Institute of Thessaloniki, Greece, \\ Email driordanis@yahoo.gr
}

Received: March 17, 2020 | Published: March 31, 2020

\section{Introduction}

Public swimming pools in Greece operate under the supervision of local municipalities and in some cases by the General Secretariat of Sport. They are used almost throughout the year as most of them are heated, either indoor or outdoor. The users of public swimming pools belong to two population groups. One group includes swimming athletes who are registered with a sports club and the other group concerns the public. The second group consists mainly of adults who use the pools for entertainment, well-being and sports. The second group of swimmers is clearly bigger than the first. The swimmers of this group swim all year round but mainly use the swim tanks from May to September. This is partly due to the fact that part of the general population cannot afford a summer vacation program. In this category belongs, regardless of gender and age, the unemployed and the workers who have very low salary. Families with children are not so common in public swimming pools. Swimming pool rules require that the potential swimmer registers a medical report attesting that he is in good general health for his participation in swimming and lacks potentially transmissible dermatological diseases. In particular, a medical report from a doctor of internal medicine or a General Practitioner or a Cardiologist and a Dermatologist is required. Women need extra medical report from a Gynecologist.

The Dermatologist's medical report refers to the absence of communicable diseases of the skin as well as its good consistency.
The general public is referred to physicians of their choice, while swimming athletes are usually examined by physicians of the sports club to which they belong.

Dermatological diseases that might be transmitted to other swimmers and constitute a reason for the exclusion of a potential swimmer, belong to four major groups and concern infections from fungi, viruses, bacteria and parasites. ${ }^{1}$

There are protozoan parasites which can be transmitted into the pool water such as the trichomonas. ${ }^{2}$ The aquatic and warm environment of the pool constitutes the ideal conditions for the development and transmission of certain dermatological diseases such as fungi. ${ }^{3}$ The same is true for the surrounding areas such as changing rooms and personal hygiene areas. Inhibition of the growth of these diseases is achieved by water chlorination and excellent hygiene in public areas such as changing rooms and shower rooms. ${ }^{4}$ Chlorine inhibits the growth of bacteria and the growth of viruses, fungi and parasites but not to the same extent. ${ }^{5}$ The effect of chlorine on the growth and transmission of viruses, fungi and parasites is moderate to poor. ${ }^{6}$

Fungal infections grow easier than other infections in a wet and warm environment. The common pathogenic fungal strains found in swimming pools and surrounding areas are more than 50 . The most frequently detected species are different types of Candida, Trichophyton interdigitale, Trichophyton Rubrum, Trichophyton mentanographytes and Aspergillus. ${ }^{7}$ The most common clinical forms 
of fungal infections include onychomycosis, tinea corporis, tinea cruris, tinea pedis (athlete's foot) and tinea manuum. ${ }^{8}$

The most common viral infection that can potentially be transmitted in pool areas is mainly palm and toe warts. ${ }^{9}$ The second most common transmitted viral skin infection is Molluscum contagiosum. ${ }^{10}$ Molluscum contagiosum as a disease is highly contagious. ${ }^{11}$ The most common viral infections most often transmitted into the pool water are not the dermatological ones, but the ones involving the gastrointestinal system and especially the enteroviruses. This type of infection manifests quiet often with diarrhea.

A bacterial infection that is a cause of exclusion from using swimming pools is the impetigo which is highly contagious and is due to Staphylococcus or Streptococcus. There are bacterial infections that are more commonly detected in pool water and are related to Pseudomonas and Eschericia Coli. ${ }^{12}$ The infection from pseudomonas is capable of causing folliculitis. ${ }^{13}$ It can also manifest as dermatitis of the outer ear. ${ }^{14}$ Scabies are transmitted mainly in the changing rooms rather than in the pools. This is due to the towels been deposited by different swimmers on the benches.

The clinical picture of each infection can vary from person to person and can present difficult diagnostic problems to the physician who is going to provide a medical report. One of the most common differential diagnostic problems is the warts that may be misdiagnosed as corns. Quite often a plantar fungal infection can be considered as eczema or contact dermatitis, which is also a very common problem in differential diagnosis.

Moreover, the true clinical picture may be altered or even concealed by preceding incomplete treatments. Patients often use medication of their own choice without taking a medical advice by a physician. It is a frequent phenomenon and usually refers to diseases that according to the patient seem to be minor or not directly threaten his or her general health. Dermatology involves not only the detection of transmissible diseases but also the detection of areas of the skin where there may be a disorder in its consistency. The presence of an open wound can more easily lead to infection of the skin since in this case the affected skin acts as an entrance gate. ${ }^{15}$ There is not strong evidence that the protective cover of an infected skin area is adequate to avoid the transfer of the infection. ${ }^{16}$

In Greece at the recent years due to serious economic problems a significant part of the general population is using public swimming pools because it cannot afford the cost of summer vacations. It should be noted that more than $70 \%$ of the Greek population resides less than 50 kilometers from the sea. Also, due to the adverse financial parameters, most of the prospective swimmers do not go to the specialized Dermatologist for medical report regarding transmissible dermatological diseases, but they receive this report from a General Practitioner or internal medicine doctor. The family doctor will give otherwise the medical report consulted their general state of health in order to be able to swim. It should be noted that dermatological diseases number around 2000 diseases together with their various variants and subcategories. ${ }^{17}$ At the same time, it is sometimes difficult to distinguish them not only for doctors of other specialties but also for dermatologists themselves.

It was therefore considered appropriate to investigate the accuracy of the medical reports given by other specialties on dermatological diseases.

\section{Methods}

The study was conducted during spring and summer, where most of the swimmers are enrolled in public swimming pools. Specifically, the study took place from the beginning of May 2015 until the end of September 2015 at the Municipal Swimming Pool of the Metropolitan Municipality of Thessaloniki, Greece.

The candidate swimmers were examined at the swimming pool's first aid medical office in order to exclude any dermatological diseases that could possibly be transmitted to other swimmers. They were then given a medical report that they were healthy or in case a dermatological disease was found, they were given an appropriate treatment and were advised to be re-examined in order to receive a medical report that allow them access to the pool. Only after the successful treatment and complete remission of their dermatological disease they were allowed to swim.

The clinical examination was provided by the dermatologist free of charge. Participation was voluntary and only the swimmers who wished to be examined were included. All of the examined people were adults. The swimming pool regulation states that children who want to swim should be accompanied by an adult. The medical report for the children is provided by the Pediatrician who certifies the child's overall health.

A total of 1090 people were examined, of which 573 were women and 517 were men of all ages. The 479 people who were examined in the swimming pool's medical office had no medical report certifying that they did not suffer from a transmissible dermatological disease or they had only a medical report from a doctor of internal medicine or from a General Practitioner referring to the general health profile of the nominated swimmer without any reference to the dermatological skin diseases.

The rest 611 people had recent (maximum one week) medical report from a General Practitioner or a doctor of internal medicine certifying that they did not suffer from a communicable dermatological disease and were in good physical health for performing the swimming exercise (Figure 1).

\section{Examinated per specialty}

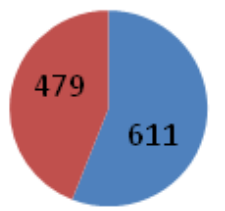

Family Doctors

- Dermatologist

Figure I People examined per medical specialist.

This second group was re-examined voluntarily by a dermatologist in order to renew for free the duration of their recent (maximum one week) medical report, which was given to them by another specialty.

It is worth noting that the validity of all medical reports is six months for swimmers over 65 years of age and one calendar year for those swimmers who are under 65 years of age. The clinical dermatological examination included an examination of the palms, soles, and later the trunk (anterior and posterior surfaces) as well as the limbs and the face. The scalp area and the perinatal area were not examined as there was mandatory coverage within the pool. 
The work was conducted in accordance with the COPE of Ethics of the World Medical Association (Declaration of Helsinki) for studies involving humans. Written informed consent was obtained from all patients. The study was approved by the Department of the Medical Services of the Municipality of Thessaloniki. Data was analyzed by Statistical package of Social Science version (SPSS). The considered statistical significance was $\mathrm{p}<0.05$.

\section{Results}

The first group of the examined swimmers consists of those with no dermatological report that were 479 people in total, of whom 263 were women and 216 were men. Patients with communicable dermatological disease were 56 of whom 30 were women and 26 were men. The dermatological diseases identified were mainly viral and fungal infections and less bacterial and parasitic infections. In total $11.7 \%$ of the examined people of this group were affected. That is, $12 \%$ of men and $11.4 \%$ of women suffered from a dermatological disease. There was virtually no significant difference in the dermatological diseases that affected men and women.

Viral infections represented mostly by the HPV group virus and lesions were found in the palms and soles of patients. Nineteen patients presented with an HPV infection accounted for about $4 \%$ of all subjects of this group. Most patients had only one lesion with a major locus in palms or soles. Few patients had multiple wart lesions.
More pathological findings were manifested to the soles regarding to the palms.

The fungal infections were related with the candida or with different types of dermatophytes..$^{18}$

The clinical findings were mostly onychomycosis, athlete's foot and cutaneous candidiasis. The total number of patients with fungal infections was 29 , or about $6 \%$ of all examined people of the group. ${ }^{19}$

Specifically, 14 patients had onychomycosis, almost $3 \%$ of all subjects and most of them had fungal infection between the toes of the legs. Most onychomycosis affected the nail of the big toe of the foot.

The remaining forms of fungal infections included 15 patients without nail infiltration. These fungi affected not only the upper and lower limbs but also the trunk.

Bacterial infections were mainly manifested as furuncle, folliculitis and impetigo and affected 8 people, or about $2 \%$ of those examined. The cause of the folliculitis was not only staphylococcus but fungi too. The main location of these diseases was in the trunk.

There were also patients with parasitic but non-communicable findings such as mosquito bites or other insects. No patient was found to be suffering from transmissible parasitic diseases such as body lice or scabies (Figure 2).

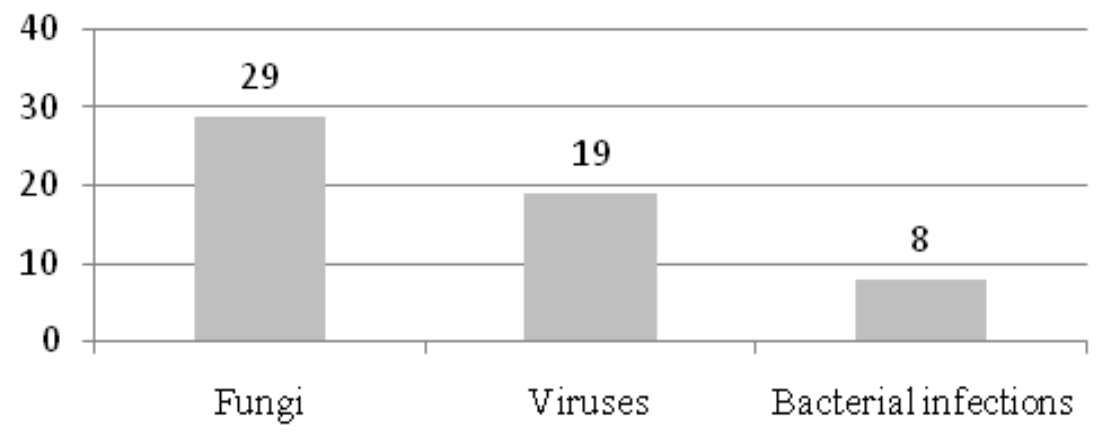

Figure 2 Causative agents of dermatological diseases of the first group.

The second group included people who had $\alpha$ recent medical report, regarding their dermatological condition, given by a General Practitioner or an internal medicine doctor. There were totally 611 people in this group of whom 342 were women and 269 were men.
Patients with a dermatological disease that might be transmitted to other swimmers were 35 out of which 19 were women and 16 were men (Figure 3).

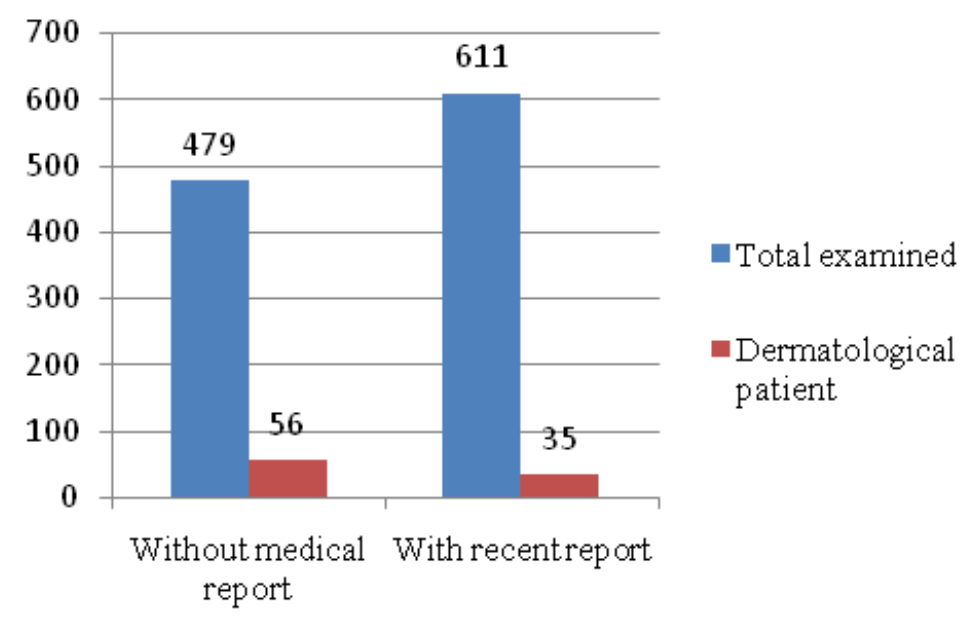

Figure 3 Total of examined swimmers and patients by medical specialty. 
In total, $5.7 \%$ of the patients of this group who had already been examined by a family doctor manifested a dermatological disease. Virtually one out of two swimmers of this group suffered from a potentially contagious dermatological disease although he was already characterized as healthy by a General Practitioner or internal medicine doctor. The infections concerned only warts and fungi in the area of the palms, the soles and in the trunk (Figure 4).

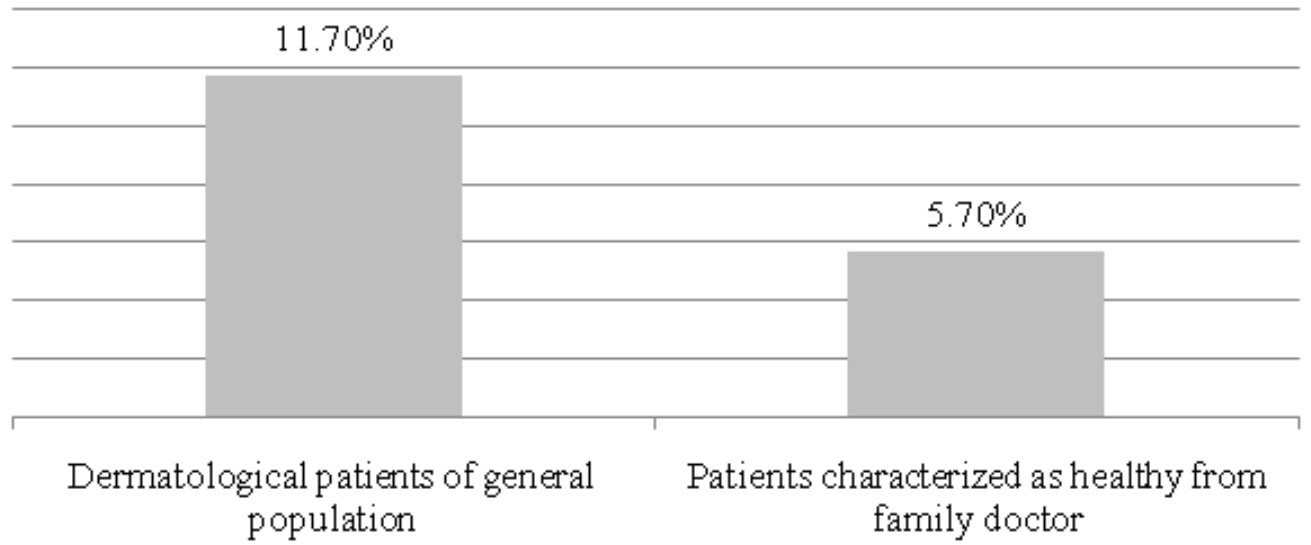

Figure 4 Dermatological patients of the general population and dermatological patients identified as healthy by a family physician.

Patients suffered from palm or toe warts and who had already been examined by a family doctor were 16 while those who suffered from onychomycosis or other fungal infection were 19 . No swimmer was found with a bacterial or parasitic infection or with an open wound.

In the first group, the sufferers from warts were 19, almost the $4 \%$ of the examined people, while in the second group the sufferers from warts were 16 , which is $2.6 \%$ of the examined people. The people with warts of the second group manifested one or two lesions. Apparently swimmers with more than two lesions were excluded by the General Practitioner or the internal medicine doctor since in the case of multiple warts lesions, the diagnosis is easier. On the contrary, isolated lesions can be misdiagnosed as a result of increased mechanical pressure in the upper extremities due to manual labor and in the lower extremities due to the use of footwear.

In the first group, 29 patients presented a fungal infection and accounted for $6 \%$ of the people examined, whereas in the second group 19 patients had fungal infection, thus the $3.1 \%$ of the examined people. It is recalled that the second group had been diagnosed as dermatologically healthy by a family doctor.

Most of the patients with warts in the second group were diagnosed as healthy, while about half of those suffering from various fungi infections were also found to be healthy (Figure 5).

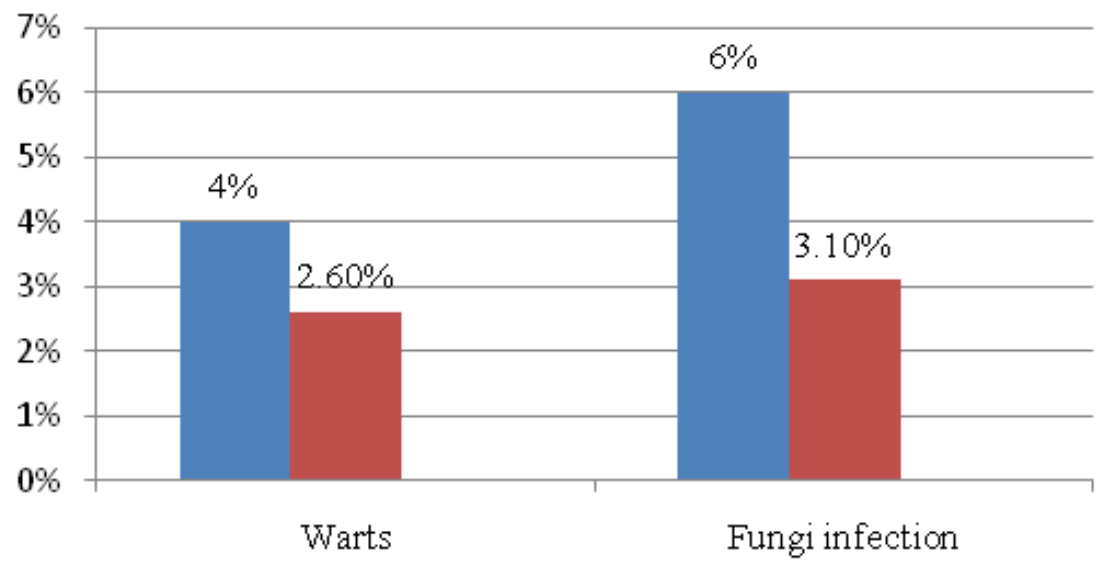

Figure 5 Dermatological diseases between the two groups.

\section{Conclusion}

Obviously there are diagnostic issues related to potentially transmissible dermatological diseases when the examination is done not by a specialized dermatologist but by a general practitioner or an internal medicine doctor. ${ }^{20}$ The medical report granted that a swimmer can use a public swimming pool concerns the exclusion of potentially transmissible dermatological diseases to other swimmers.
This certification is important because it notifies the person of the presence or absence of a dermatological disease and, in addition, protects the public from the transmission of such diseases. It also reduces the cost of medical expenses that would be required to cover those diseases that might have been transmitted to other swimmers.

The decline in health care cost also refers to the sufferer himself, as both fungi and warts tend to spread and complicate as long as they are left untreated. 
In particular, fungi, if detected at the beginning of the onset of skin attack, may possibly be treated using only topical treatment. In later stages, fungal disease will also require per os medication. This greatly increases the overall cost of treatment.

The reasons for the incomplete diagnosis by General Practitioners and internal medicine doctors are identified in various fields such as the nature of dermatological diseases and the training of other specialties in dermatology. ${ }^{21}$

Besides due to the difficult Greek economic situation, the majority of the population chooses to visit a doctor who will certify that they are healthy from both a dermatological and a physical aspect. Most people, perhaps considering that they are already healthy, do not consider it advisable to see a specialist. Many times an early dermatological condition may not disrupt the patient's daily routine and may have mild symptomatology.

However, in cases of contagious dermatological diseases, the initial poor clinical picture allows the disease to spread to the patient as well as to the rest of the population. The sufferer of warts or fungi upon entry into the pool is likely to aggravate his dermatological problem. Also the infection with warts is very possible to be transmitted to other swimmers.

The contagious dermatological infections can easily spread to pool areas such as showers and changing rooms. In this case, the financial cost of the treatment increases according to the severity of the clinical picture.

The clinical picture of most dermatological diseases is often indistinguishable, so that even dermatologists themselves use further diagnostic tools such as dermatoscopy to certify the diagnosis of a disease and to solve differential diagnostic issues. ${ }^{22}$ Short-term education to these diagnostic techniques will be very useful. ${ }^{23}$ Since the volume of dermatological diseases is so high, more and more targeted training is needed for general practitioners on these diseases. ${ }^{24}$ It would be advisable for this customized education to initially focus on the most widely occurred dermatological diseases. ${ }^{25}$ Continuous training on issues related to the diagnosis of dermatological diseases through seminars during the professional life of a family doctor is considered necessary. ${ }^{26}$

Unfortunately, medical report regarding thepool entry refers mainly to public pools and not private ones such as hotels and spa. In these areas, the risk of transmit ion of a dermatological disease to swimmers is more likely due to a lack of medical control of swimmers' ability to access private swimming pools regardless of whether water is treated properly and hygiene rules are followed in the surrounding environment. ${ }^{27}$

There are usually posted warning signs indicating that the swimmers should be in good physical health and not suffering from contagious dermatological diseases or open wounds. Obviously, since dermatological diseases that can be transmitted through swimming pool water are identified to swimmers who already had a clinical examination by the General Practitioner or an internal medicine doctor, it is certain that the corresponding diseases are also referred to a greater extent to prospective swimmers who have not been examined by a Dermatologist or a family doctor. It is advisable to change the scope of the valid regulations for the allowance of a person to enter a pool, whether it is public or private, in order to protect the public health.
The results of this study highlight the different diagnostic difficulty that other medical specialties may have when they are called upon to recognize dermatological diseases.

It is quite possible that most patients with dermatological symptoms are initially referred to the family physician rather than to a specialized dermatologist. Given the large volume of dermatological diseases, it can be said that this may conceal a greater degree of loss of dermatological diagnoses by family physicians. Similar situations of loss of diagnosis by family doctors might also be found in diseases referring to other medical specialties. ${ }^{28}$ Not only the better education of family physicians on dermatology, but also the close cooperation between them and dermatologists, is considered to be imperative.

\section{Conflicts of interest}

The author declares that there is no conflicts of interests.

\section{Acknowledgments}

None.

\section{Funding}

None.

\section{References}

1. El Baze P, Ortonne JP. Les infections et les dermatosesacquisesdans les piscines. Ann Dermatol Venereol. 1991;118:973-977.

2. Pereira-Neves A, Benchimol M. Trichomonasvaginalis: in vitro survival in swimming pool water samples. Exp Parasitol. 2008;118(3):438-441.

3. Basler RSW, Basler DL, Basler GC, et al. Cutaneous injuries in women athletes. Dermatol Nurs. 1998;10:9-18.

4. Koehn GG. Skin injuries in sports medicine. J Am Acad Dermatol. 1991;24(1):152.

5. Basler RS, Basler GC, Palmer AH, et al. Special skin symptoms seen in swimmers. J Am Acad Dermatol. 2000;43:299-305.

6. Sarnaik AP, Vohra MP, Sturtman SW et al. Medical problems of the swimmer. Clin Sports Med. 1986;5:47-64.

7. Ekowati Y, Ferrero G, Kennedy MD, et al. Potential transmission pathways of clinically relevant fungi in indoor swimming pool facilities. International Journal of Hygiene and Environmental Health. 2018;221(8):1107-1115.

8. Shemer A, Gupta AK, Amichai B, et al. Increased Risk of Tinea Pedis and Onychomycosis among Swimming Pool Employees in Netanya Area, Israel. Mycopathologia. 2016;181(11-12):851-856.

9. Al-Mutairi N, AlKhalaf M. Mucocutaneous warts in children: clinical presentations, risk factors, and response to treatment. Acta Dermato Venereol Alp Pannonica Adriat. 2012;21(4):69-72.

10. NiizekiK, Kano O, Kondo Y.An epidemic study of molluscumcontagiosum. Relationship to swimming. Dermatologica. 1984;169(4):197-198.

11. Choong KY, Roberts LJ. Molluscum contagiosum, swimming and bathing: a clinical analysis. Austral J Dermatol. 1999;40(2):89-92.

12. Washburn J, Jacobson JA, Marston E et al. Pseudomonas aeruginosa rash associated with a whirlpool. JAMA. 1976;235(20):2205-2207.

13. Yu Y, Cheng AS, Wang $\mathrm{L}$,et al. Hot tub folliculitis or hot hand-foot syndrome caused by Pseudomonas aeruginosa. J Am Acad Dermatol. 2007;57(4):596-600. 
14. Lutz JK, Lee J. Prevalence and antimicrobial-resistance of Pseudomonas aeruginosa in swimming pools and hot tubs. Int $J$ Environ Res Public Health. 2011;8(2):554-564

15. Penso-Assathiany D, Flahault A, Roujeau JC. Warts, swimming pools and atopy: a case control study conducted in a private dermatology practice. Ann DermatoloVenereol. 1999;126(10):696-698.

16. Vaile L, Finlay F, Sharma S. Should verrucas be covered while swimming? Arch Dis Child. 2003;88(3):236-237.

17. DA Burns, NH Cox. Introduction and Historical Bibliography: The dermatologist's work. In: T. Burns, S Breathnach, N. Cox, et al, Rook's Textbook of Dermatology. Oxford, Blackwell Science Ltd. 2004:1.3

18. Jankowski M, Charemska A, Czajkowski R. Swimming pools and fungi: An epidemiology survey in Polish indoor swimming facilities. Mycoses. 2017;60(11):736-738.

19. Gudnadóttir G, Hilmarsdóttir I, Sigurgeirsson B. Onychomycosis in Icelandic swimmers. Acta Derm Venereol. 1999;79(5):376-377.

20. Richard MA, Joly P, Roy Geffroy B, et al. Public perception of dermatologists in France: results from a population-based national survey. J Eur Acad Dermatol Venereol. 2019;33(8):1610-1615.

21. Ramdas K, van Lee C, Beck S, et al. Differences in Rate of Complete Excision ofBasal Cell Carcinoma by Dermatologists, Plastic Surgeons and General Practitioners: A Large Cross-Sectional Study. Dermatology. 2018;234(3-4):86-89.
22. Jones OT, Jurascheck LC, Utukuri M, et al. Dermoscopy use in UK primary care: a survey of GPs with a special interest in dermatology. $J$ Eur Acad Dermatol Venereol. 2019;33(9):1706-1712.

23. Augustsson A, Paoli J. Effects of a 1-Day Training Course in Dermoscopy Among General Practitioners. Dermatol Pract Concept. 2019;9(3):195199.

24. Marasca C, Annunziata MC, Cacciapuoti S, et al. A Dermatological Questionnaire for General Practitioners with a Focus on Hidradenitis Suppurativa. Open AcessMaced J Med Sci. 2018;6(10):1902-1905.

25. Lopes S, Vide J, Costa-Silva M, et al. Awareness, knowledge, and practice patterns of general practitioner residents and specialists toward hidradenitis suppurativa: a survey study. Acta DermatolVenereol Alp Pannonica Adria. 2019;28(2):61-63.

26. Beecher SM, Keogh C, Healy C. Dedicated general practitioner education sessions can improve diagnostic capabilities and may have a positive effect on referral patterns for common skin lesions. Ir J Med Sci. 2018;187(4):959-963.

27. Hopkins RS, Abbott DO, Wallace LE. Follicular dermatitis outbreak caused by Pseudomonas aeruginosa associated with a motel's indoor swimming pool. Public Health Rep. 1981;96(3):246-249.

28. Clare R Goyder, Caroline HD Jones, Carl J Heneghan, et al. Missed opportunities for diagnosis: lessons learned from diagnostic errors in primary care. British Journal of General Practice. 2015;65(641):e838e844. 